\title{
Extension of the ECRH operational space with O2 and X3 heating schemes to control tungsten accumulation in ASDEX Upgrade
}

\author{
H. Höhnle ${ }^{1}$, J. Stober ${ }^{2}$, A. Herrmann ${ }^{2}$, W. Kasparek ${ }^{1}$, \\ F. Leuterer ${ }^{2}$, F. Monaco ${ }^{2}$, R. Neu ${ }^{2}$, D. Schmid-Lorch ${ }^{2}$, \\ H. Schütz ${ }^{2}$, J. Schweinzer ${ }^{2}$, U. Stroth ${ }^{1,2}$, D. Wagner ${ }^{2}$, \\ S. Vorbrugg 2 , E. Wolfrum ${ }^{2}$ and the ASDEX Upgrade Team \\ ${ }^{1}$ Institut für Plasmaforschung, Universität Stuttgart, Stuttgart, Germany \\ ${ }^{2}$ Max-Planck-Institut für Plasmaphysik, EURATOM-Association, Garching, \\ Germany \\ E-mail: hoehnle@ipf .uni-stuttgart.de
}




\begin{abstract}
ASDEX Upgrade is operated with tungsten-coated plasma-facing components since several years. H-mode operation with good confinement has been demonstrated. Nevertheless purely NBI-heated H-modes with reduced gas puff, moderate heating power or/and increased triangularity tend to accumulate tungsten, followed by a radiative collapse. Under these conditions, central electron heating with $\mathrm{ECRH}$, usually in X2 polarisation, changes the impurity transport in the plasma centre, reducing the central tungsten concentration and, in many cases, stabilizing the plasma. In order to extend the applicability of central ECRH to a wider range of magnetic field and plasma current additional ECRH schemes with reduced single pass absorption have been implemented: X3 heating allows to reduce the magnetic field by $30 \%$, such that the first H-modes with an ITER-like value of the safety factor of $q_{95}=3$ could be run in the tungsten-coated device. $\mathrm{O} 2$ heating increases the cutoff density by a factor of 2 allowing to address higher currents and triangularities. For both schemes scenarios have been developed to cope with the associated reduced absorption. In case of central X3 heating, the X2 resonance lies close to the pedestal top at the high-field side of the plasma, serving as a beam dump. For O2, holographic mirrors have been developed which guarantee a second pass through the plasma centre. The beam position on these reflectors is controlled by fast thermocouples. Stray-radiation protection has been implemented using sniffer-probes.
\end{abstract}

PACS numbers: $52.50 . \mathrm{Sw}, 52.25 . \mathrm{Vy}$

Submitted to: Nucl. Fusion

\title{
1. Introduction
}

The ECRH system at ASDEX Upgrade consists of four "old" gyrotrons at $140 \mathrm{GHz}$, each delivering a power of $0.5 \mathrm{MW}$ over $2 \mathrm{~s}$, and a new system, which is currently being installed. The new ECRH system will consist of four multi-frequency gyrotrons delivering $1 \mathrm{MW}$ over a complete ASDEX Upgrade discharge (10 s) [1, 2]. The typically used second harmonic extraordinary mode (X2 mode) at $2.5 \mathrm{~T}$ has the advantage of a complete and localized absorption. These two systems were especially designed for transport studies with local electron heating and MHD control with electron cyclotron current drive. An additional application arises when the first wall material at ASDEX Upgrade was changed from carbon to the high $\mathrm{Z}$ material tungsten. In improved Hmodes with low gas puff the plasma tends to accumulate tungsten $[3,4]$, followed by a radiation collapse. In such discharges central ECRH (inside the $q=1$ surface) leads to a change in the electron temperature gradient and to a modification of the turbulent transport triggering an outward flux of the impurities $[5,6,7,2]$.

However, the usage of central X2 heating with $140 \mathrm{GHz}$ in the plasma centre requires $B_{t}(0) \approx 2.5 \mathrm{~T}$ and $n_{e}(0)<1.2 \cdot 10^{20} \mathrm{~m}^{-3}$ (X2 cutoff). The latter is in conflict with the requirement of ITER-like discharges to be operated at a safety factor of $q_{95} \approx 3$ at $70-90 \%$ of the density limit $n_{G W}>1.7 \cdot 10^{20} \mathrm{~m}^{-3}$ (for $I_{P}>1.3 \mathrm{MA}$ ). The X2 cutoff limits $\mathrm{H}$-mode operation with central heating close to this density limit to $q_{95}>4.5$ 
depending on plasma shape otherwise the stray radiation would increase. To protect in-vessel components from ECRH stray radiation when operating the ECRH close to the cutoff, sniffer probes [8] were installed at ASDEX Upgrade, detecting the non absorbed microwave power. The setup and some measurements with these probes are discussed in section 4 . With these probes a real-time interlock is realized to switch off the ECRH when reaching a cutoff. Here two schemes are presented which circumvent the limitation of ECRH for ITER-like H-mode operation.

One approach to avoid cutoffs at $q_{95} \approx 3$ is to reduce the magnetic field by $30 \%$, where the third harmonic extraordinary mode (X3 mode) is absorbed in the core. However, this mode suffers from incomplete absorption [9] at electron temperatures of $\approx 3 \mathrm{keV}$. This heating scenario is described in section 2.1 and the experimental results are presented in section 5.1.

An alternative is to use the ordinary mode (O2 mode) at the second harmonic of the resonance at $B_{t}(0)=2.5 \mathrm{~T}$. This mode has a cutoff at the plasma frequency, which is equivalent to twice the cutoff density of the X2-mode cutoff. But the disadvantage of the $\mathrm{O} 2$ mode is also an incomplete absorption [9] at typical electron temperatures of $3-4 \mathrm{keV}$. To increase the optical thickness $\tau \propto T_{e}^{2}[10]$, a mirror for a second pass of the beam through the plasma is mounted at the inner column of ASDEX Upgrade. The design of the mirror and the associated heating scenario are described in section 3 and 2.2 , respectively. First experimental results with a second pass of the beam are shown in section 5.2 and 5.3. Finally, the paper is summarized and the results are discussed in section 6 .

\section{Heating Scenarios}

To develop new ECRH scenarios in high density plasmas, or at lower magnetic fields, the beam tracing code TORBEAM [11] was used, which calculates the ray trajectory, the deposition and the current drive profiles of the ordinary and extraordinary beam for different injection angles, magnetic configurations, density and temperature profiles.

\subsection{X3-Heating Scenario}

For the X3-heating scenario a central deposition of the ECR power is realized at a toroidal field of $B_{t}(0) \approx 1.7 \mathrm{~T}$. At this field, a $q_{95}$ of about 3 is achieved at a plasma current of $1 \mathrm{MA}$. But for the accessible plasma parameters in ASDEX Upgrade the absorption is incomplete. To handle the shine-through power the magnetic field is increased to $1.8 \mathrm{~T}$; thereby the resonances are shifted to the low field side such that the $\mathrm{X} 2$ resonance is shifted from the scrape of layer on the high field side to the pedestal top (Fig. 1 left), where the shine-through is completely absorbed. In this scenario the X3 resonance is shifted by a few $\mathrm{cm}$ from the axis, but the absorption at the $\mathrm{X} 3$ resonance is still central enough to avoid tungsten accumulation. The TORBEAM simulation (Fig. 1 ) shows a central absorption of $\approx 70 \%$ at $r / a<0.2$, which is equal to $q<1$ (see also 

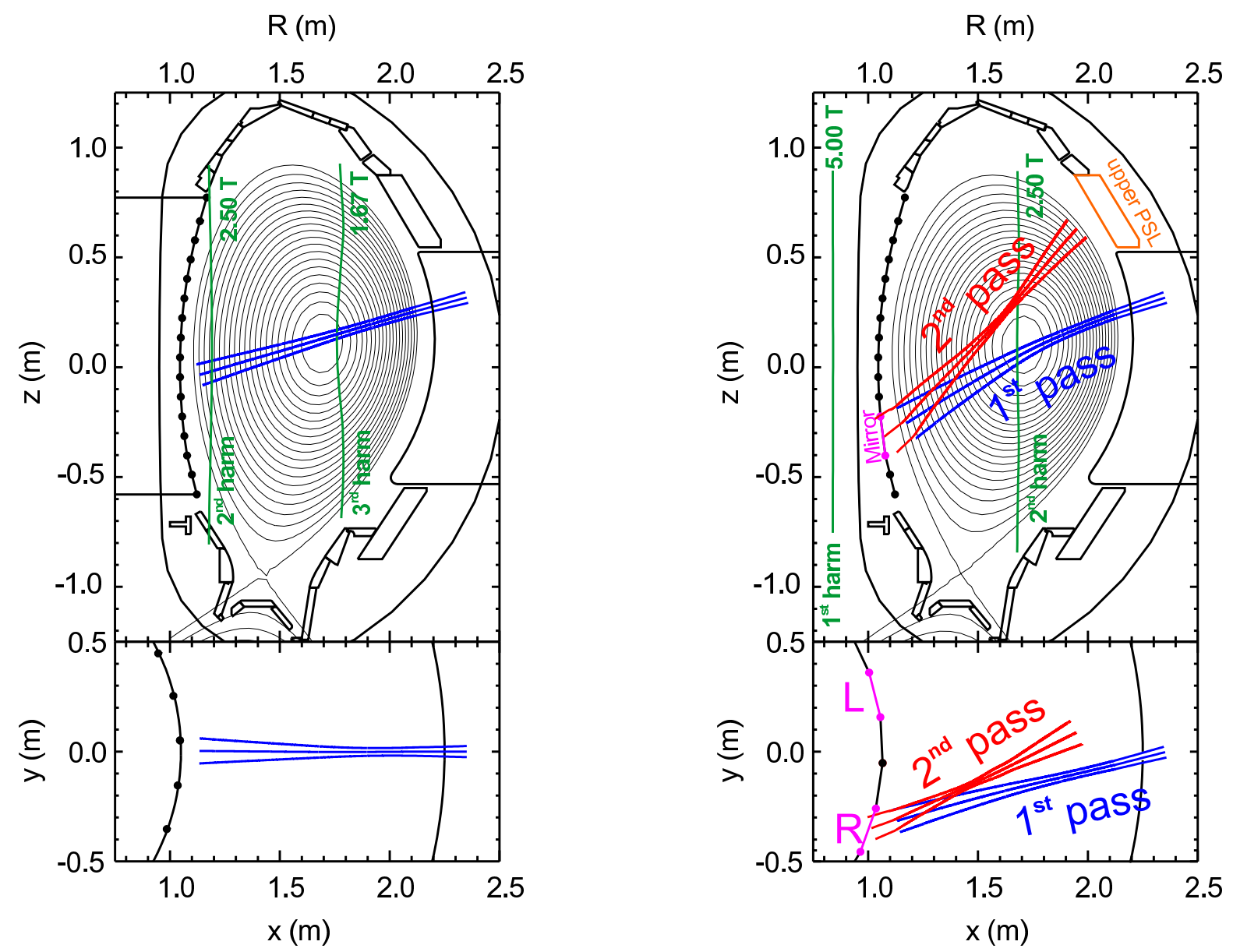

Figure 1. Poloidal and toroidal cross section of ASDEX Upgrade with resonant layers and injection paths calculated by TORBEAM. Left: X3 heating, where the non absorbed ECRH power $(\approx 30 \%)$ at the third harmonic resonance is completely absorbed at the second harmonic located at the high field side. Right: the O2-heating scenario with the beam launched from the new steerable launcher, hitting after a first pass through the plasma centre (75\% is absorbed) the holographic mirror at the inner column of ASDEX Upgrade. Here the beam is reflected and refocused back through the core in the direction of the upper PSL (passive stabilization loop).

Ref. [12]) for realistic plasma parameters of $n_{e}(0)=1.0 \cdot 10^{20} \mathrm{~m}^{-3}$ and $T_{e}(0)=2.5 \mathrm{keV}$. The functionality of the beam dump was demonstrated in comparison experiments at a magnetic field of $1.8 \mathrm{~T}$ (with beam dump) and $1.67 \mathrm{~T}$ (without beam dump). At the lower magnetic field the stray radiation was significantly higher as in the higher field case with the associated beam dump [13].

A dedicated local effect of the ECRH deposition at the X2 resonance on the performance of the plasma edge is not seen in experiments varying the magnetic field slightly. This is not surprising since the ECRH power deposited just inside the pedestal top (here $\approx 300 \mathrm{~kW}$ at $1 \mathrm{MW}$ injected power) is significantly smaller than the total heat flux at this location (here $\approx 7 \mathrm{MW}$ ) and comparable to the power deposited by the NBI between this location and the separatrix, which is $5-10 \%$ of the total NBI power 
(here $\approx 500 \mathrm{~kW})$ as estimated by the NUBEAM code $[14,15]$. This NBI heating at the $\mathrm{H}$-mode edge is also preferential electron heating due to the relatively low electron temperatures in this region of the plasma.

\subsection{O2-Heating Scenario}

To investigate the O2-heating scenario with the TORBEAM code, expected plasma parameters for a 1.4 MA discharge were chosen $\left(n_{e}(0)=1.4 \cdot 10^{20} \mathrm{~m}^{-3}, T_{e}(0)=3.5 \mathrm{keV}\right)$. For these parameters the theory predicts an optical thickness of approximately 2 (absorption up to $86 \%$ ) for an optimal toroidal injection angle of $\approx 15^{\circ}[9,10,16]$.

The TORBEAM calculations were done for different poloidal $(\theta)$ and toroidal $(\phi)$ injection angles for the new steerable launcher [1]. The absorbed power for the different angles is plotted in Fig. 2 as a contour plot. The bright and dark colours are linked to regions with high and low absorption, respectively. As expected, the highest absorptions of $\approx 80 \%$ can be achieved at around $\phi=15^{\circ}$. Because of this incomplete absorption, the machine safety is not guaranteed and problems concerning local stray radiation are expected. Therefore, the non-absorbed power must be further reduced. The only possibility to rise the absorption of the ECRH beam in $\mathrm{O} 2$ mode is a second pass of the beam through the plasma. Reflectors installed at the inner column of ASDEX Upgrade can handle this problem; the design of such mirrors is described in more detail in section 3 .

The poloidal and toroidal cross section of the used configuration is shown in Fig. 1 on the right-hand side. The blue curve illustrates the first and the red the second pass of the beam through the plasma. The violet region at the inner wall of ASDEX Upgrade shows the location of the holographic mirrors. The injection angles were selected for good absorption and central hit on the mirrors, therefore they differ slightly from the optimal injection angle. Nevertheless, the absorption can be increased up to $94 \%$

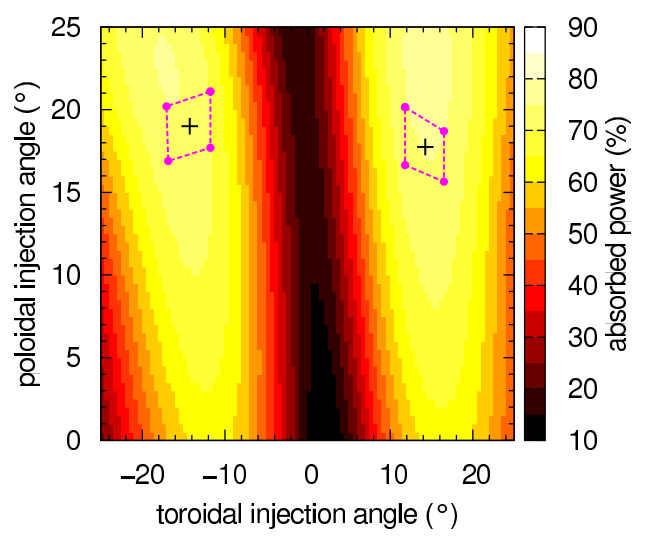

Figure 2. Absorption of O2-mode heating at ASDEX Upgrade with $n_{e}(0)=$ $1.4 \cdot 10^{20} \mathrm{~m}^{-3}, T_{e}(0)=3.5 \mathrm{keV}$ obtained from TORBEAM calculations for different toroidal and poloidal injection angles. The crosses show the central hit of the beam with the holographic mirrors (dimensions in violet lines). 
instead of $75 \%$ at single pass. The injection angles with the central hit of the beam on the mirrors are marked as crosses in Fig. 2, also the dimensions of the mirrors are shown as violet lines. A second reflection occurs on the passive stabilisation loop, where no sensitive components are mounted. The stray radiation will be further reduced when passing a third time through the plasma, but this has not been analysed numerically.

It should be mentioned that the advantage of the second pass with the holographic mirrors cannot be used in the X3-mode heating scenario at lower magnetic field. The $\mathrm{X} 2$ resonance, acting as beam dump, would have to be moved behind the mirror by decreasing the magnetic field to $B_{t}(0) \approx 1.5 \mathrm{~T}$, otherwise a fraction of the ECRH power would be absorbed in front of the mirror and could destroy it. In this case the absorption would be too far off axis and a second pass of the beam would lead to a total absorption of $\leq 60 \%$ only, which is smaller than in the X3-heating scenario described in section 2.1 .

\section{Design of the Mirror}

As described in section 2.2, a second pass of the O2-mode beam should be realized by mirrors at the inner wall of ASDEX Upgrade. These reflectors must fulfil some conditions which are essential for the safe operation in ASDEX Upgrade:

(i) The mirrors must be conformal to the inner wall, to not suffer from erosion and disturb or pollute the plasma

(ii) The mirrors have to be polarization independent, which means that the incoming O-mode beam is also reflected as beam in ordinary polarization, i.e. no phase shift between polarisation $\|$ and $\perp$ with respect to the grooves

(iii) The reflection must be directed and refocused to the plasma core to achieve an optimal second absorption $\left(\approx 15^{\circ}\right.$ toroidal injection $)$

(iv) The mirror must be manufactured out of graphite with a tungsten coating consensually with the tungsten program at ASDEX Upgrade

(v) The reflectivity of the mirror must be high $(\geq 90 \%)$ in the optimal direction and polarisation

These terms are fulfilled for holographic or phase grating mirrors. To design such gratings, the phase fronts as well as the incoming and outgoing angles of the beams on the surface of the mirrors have to be extracted from the TORBEAM simulations. With the shape of the wall tiles and the phase of the beams the pattern of the grooves can be calculated. To ease the manufacturing and the later operation some more conditions must be fulfilled:

(vi) The curvature of the grating profile has to be smooth in order to avoid high electric fields on the mirror surface and erosion of sharp structures

(vii) The milling tool limits the maximal slope angles of the grating profiles 

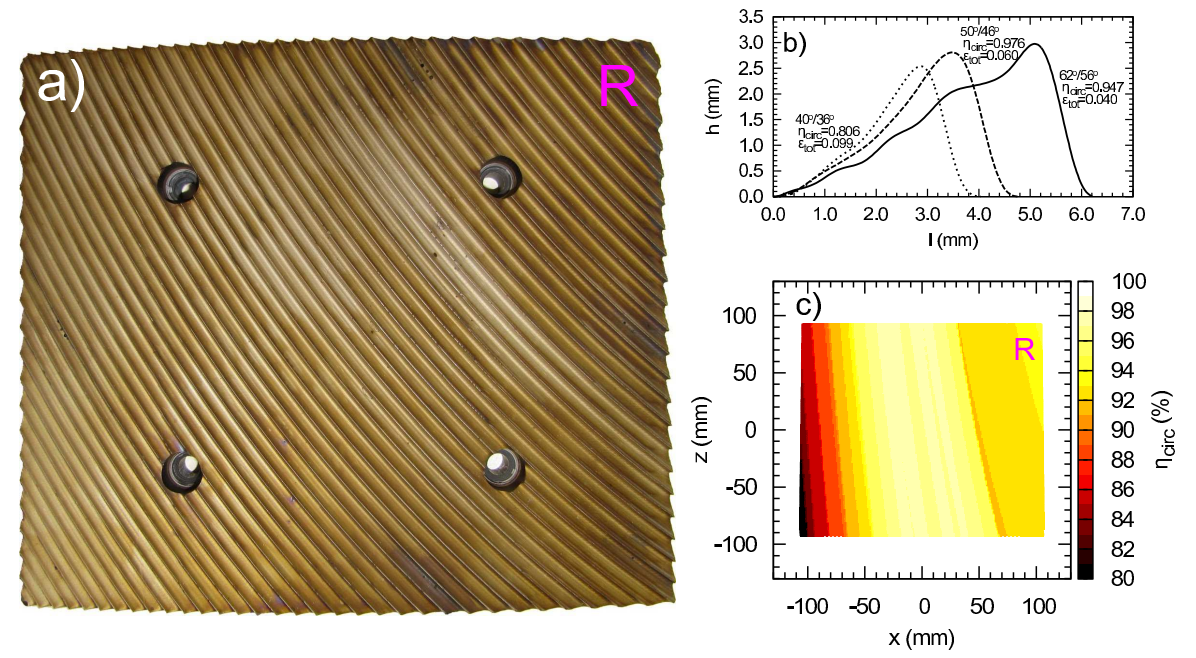

Figure 3. Photography of the holographic mirror (Fig. (a)) and sample profiles on the reflector for different incoming/outgoing angles (measured from the horizontal line) of the beam (Fig. (b)). The period length $l$ of the profile is fixed due to the combination of the incoming/outgoing angles and the chosen order of diffraction. In the optimization process the shape of the profiles was optimized for high reflection efficiency $\eta_{\text {circ }}$, independent of polarisation, in trade off manufacture limitations. The $40^{\circ} / 36^{\circ}$-profile and the $62^{\circ} / 56^{\circ}$-profile are located at the left and right edge of the mirror, the $50^{\circ} / 46^{\circ}$-profile in the centre of the mirror. In plot (c) the efficiency of the profiles is plotted on the mirror surface. In the centre of the mirror the efficiency is high $(>90 \%)$. In the edge $\eta_{\text {circ }}$ is reduced due to the limitations of the profile shape.

In order to design high-efficiency mirrors under these constraints, an optimization code [16], based on a differential evolution algorithm [17], was developed. The optimization process corresponds to a minimization of a cost function $\mathrm{C}$, which includes all the above mentioned conditions:

$$
C=\left(1-\eta_{\text {circ }}\right)+\underbrace{\varepsilon_{\text {curv }}+\varepsilon_{\text {angle }}}_{\varepsilon_{\text {tot }}}
$$

Here $\eta_{\text {circ }}$ is the efficiency of the grating for circular polarization, taking into account the efficiency in TE $(\|$ to grooves) and TM $(\perp$ to grooves) polarizations as well as the phase shift between these two polarizations. This efficiency is computed with a boundary element code $[16,18]$. The other two terms are geometrical factors and are combined to $\varepsilon_{t o t}$. $\varepsilon_{\text {curv }}$ is the curvature term, which is small for a flat profile and high for a sharply peaked profile. $\varepsilon_{\text {angle }}$ includes the condition of the limitation of the milling tool. During the optimization process it was found that the third order of diffraction of a grating implements all these conditions at best. Hence this order was chosen for the mirror. For each pair of angles a profile was optimized and at the end all profiles were put together on the dedicated location on the mirror. Under all these issues mirrors with $\approx 90 \%$ efficiency (see Fig. 3) could be calculated and manufactured out of graphite. The $10 \%$ loss of the efficiency is distributed in nearly the same parts into the other diffractive orders of the grating and in a change of the polarisation based on a small 
phase shifts between the TE and TM modes. Three sample profiles for different incoming and outgoing angles of the beam are shown in Fig. 3 (b). The efficiency for the $40^{\circ} / 36^{\circ}$ profile was found by the optimization algorithm due to the limitations of the milling tool and the desired flatness of the profiles. This profile is located at the edge and due to the power distribution of the beam it contributes only to a small fraction to the whole efficiency (see Fig. 3 (c)).

Another issue is to keep the high-power beams centred on the mirrors. A new beam position detection system, based on Ohmic losses of the microwave on the mirror surface, was installed. With thin NiCr-Ni thermocouples (diameter $0.25 \mathrm{~mm}$ and only a few ms rise time) it is planned to react on beam movements, caused for example by density changes, in real time by adjusting the poloidal injection angle with the fast steerable launcher [1]. A reaction time of a few $10 \mathrm{~ms}$ is foreseen for this issue, which is smaller than the expected energy confinement time $\tau_{e} \approx 100 \mathrm{~ms}$. The toroidal injection angle cannot be changed during the discharge, but TORBEAM calculations have shown, that this angle does not change that much during density changes and therefore can be fixed before the discharge. First experiments with this detection system are described in section 5.2.

\section{Sniffer probes}

Cutoffs and incomplete single-pass absorption pose potential hazards to the machine. The power density in the beam is up to $1 \mathrm{GW} / \mathrm{m}^{2}$ across an area of a few $\mathrm{cm}^{2}$. Absorbers (usually insulators) hit directly by the incident beam heat up within milliseconds. The problem is reduced by high reflection on metal surfaces and beam divergence. Still, stray radiation levels even in the range of $1-10 \mathrm{MW} / \mathrm{m}^{2}$ can damage insulators and uncooled thin steel housings when applied for a few seconds [19].

In case of an unexpected plasma behaviour, e.g. too high density or too low temperature leading to cutoffs or incomplete absorption, respectively, the local radiation impact may exceed critical levels requiring fast actions of the control and safety systems. This is only possible if reliable sensors are available.

In principle temperature and density profiles are available in real time during the discharge [20, 21], which could be used to cross-check absorption conditions. Then safe operation would only be possible allowing a large safety margin to account for uncertainties. Other issues not detected by real-time density and temperature profile measurements are mismatched polarisation or errors in launcher setting [19].

For these reasons an ECRH safety system should be based on direct measurement of the stray radiation. Two basic problems have to be addressed: first a large-scale anisotropy due to initially highly focused beams injected at a few toroidal positions and second a small-scale anisotropy due to interference effects of the mm-waves. The first can only be addressed by using a sufficiently large number of probes depending on vessel and plasma geometry (see below). The interference problem is well known from laboratory experiments with microwaves. If not taken into account properly, results 

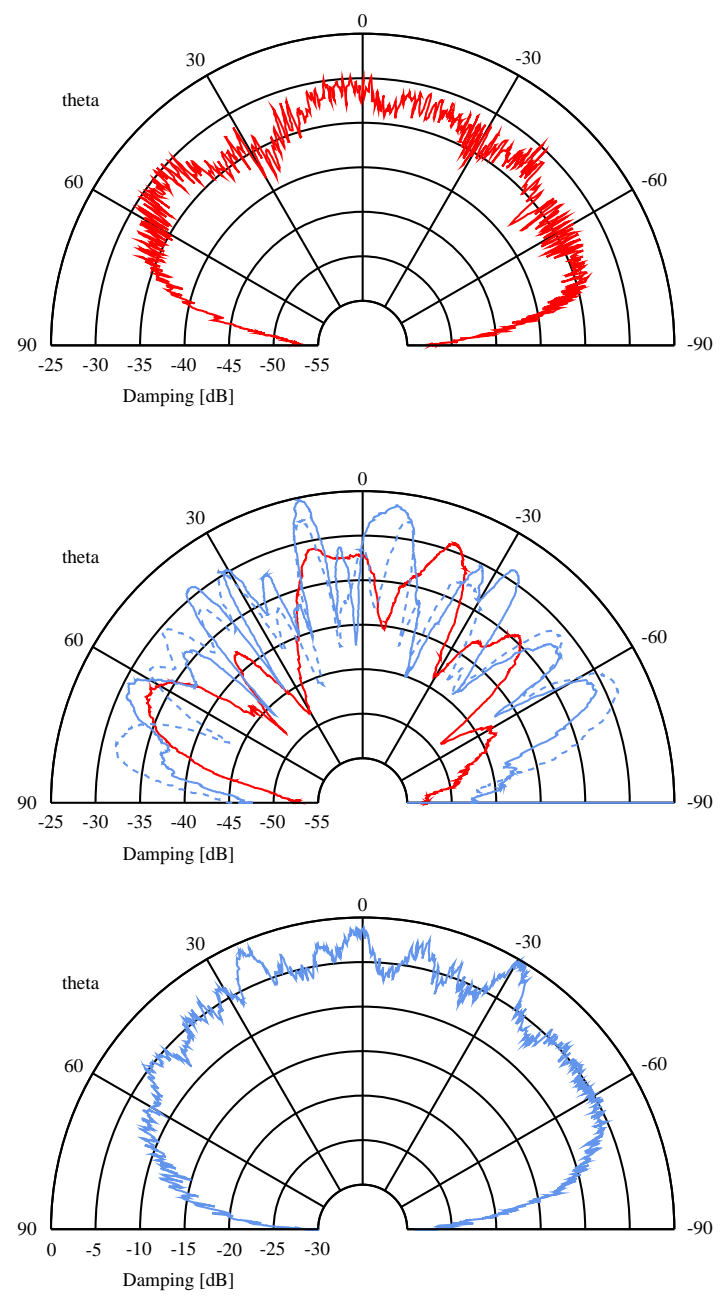

Figure 4. Detection characteristics of the sniffer probe. Top: with $10 \mathrm{MHz}$ frequency sweep, middle: without frequency sweep. In red a movable antenna driven by a BWO was used to focus a beam into the multi-mode entrance of the probe. In blue an inverse arrangement was used with an extended interaction oscillator (EIO) coupled to the mono-mode sensing exit of the probe and the antenna was used to measure the spherical power distribution exiting the sniffer-probe through its nominal entrance opening. Solid and dashed blue lines differ by a few $\mathrm{MHz}$ in EIO frequency due to drifts. Differences between red and blue curves are most likely also due to slightly different frequencies. Bottom: without frequency sweep but with a loud speaker inside the sniffer probe wrapped in Al-foil, which acts as mode mixer.

are highly erratic and useless. In Ref. [8] the attempt to overcome this problem is described using a large copper sphere (diameter: $30 \mathrm{~cm} \approx 150 \lambda$ ) connected by an overmoded waveguide (diameter: $30 \mathrm{~mm}$ ), which has a broad radiation lobe on the input (plasma) side. The sniffer probes in ASDEX Upgrade are of this type. The basic idea is to increase isotropy inside the sphere by multiple reflections and a high mode density. A diode detector is connected via a short single mode waveguide to the sphere. In the original paper the highly isotropic antenna characteristics was measured with a frequency swept source [22]. We have repeated these measurements using as a source 


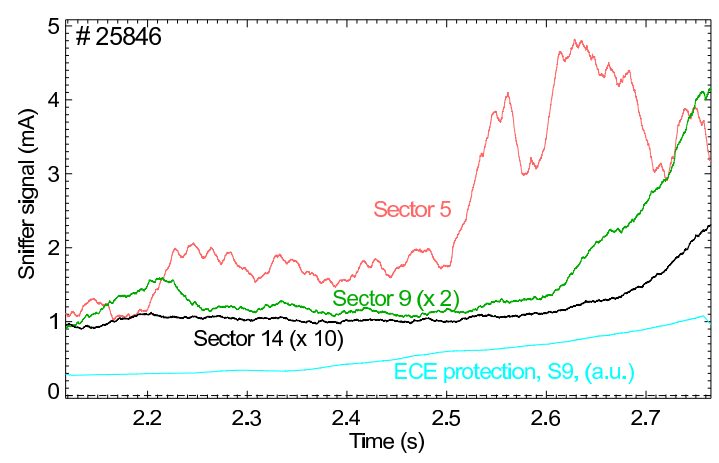

Figure 5. Smoothed signals $(30 \mathrm{~ms})$ of three different sniffer probes during a phase with $\mathrm{O} 2$ heating (see also Fig. 11 below). The signals of the probes in sectors 9 and 14 are shown on a different scale to allow better comparison of the temporal evolution. The gyrotron is located in sector 5. ASDEX Upgrade has 16 sectors. Additionally the signal from the diode protecting the ECE diagnostic is shown in arbitrary units (similarly smoothed), which is located in sector 9.

a backward wave oscillator (BWO) with and without frequency sweeping: the results with sweeping are close to those published in Ref. [8], but without sweeping poloidal asymmetries of more than $20 \mathrm{~dB}$ are found, see Fig. 4. Mode mixing by a fast moving Al-foil driven by a loud speaker mounted inside the $\mathrm{Cu}$-sphere has a similar effect as frequency sweeping.

A few $\mathrm{MHz}$ of frequency variation are sufficient to smooth the directivity of the probe, but a gyrotron has a narrow spectrum with a width of a few kHz. Still there are frequency variations in time, as for example the initial frequency chirp but also variations in the range of $1 \mathrm{MHz}$ due to the $\mathrm{AC}$ heated filament $(50 \mathrm{~Hz})$ and due to collector sweeping $(12.5 \mathrm{~Hz})$. For a fixed point in time the sniffer probe signal shows an almost white frequency spectrum (up to the $100 \mathrm{kHz}$ detection limit). The white spectrum is probably due to intrinsic plasma fluctuations, which usually show a broad frequency spectrum exceeding $100 \mathrm{kHz}$. In this picture the plasma acts as an intrinsic mode mixer for stray radiation in addition to frequency variations in time.

Figure 5 shows three sniffer probe signals from different toroidal locations for a case in which the plasma temperature dropped during O2 heating (see Fig. 11 below) using a 30 ms-average which on all three probes shows the expected rise of the stray radiation. Still the stray radiation shows a strong toroidal variation, as already reported in Ref. [8]. We note here that the sniffer signals are also highly correlated to a cutoff situation and do also rise in case of significant wrong polarisation (see Ref. [23] and next section).

The strong toroidal variation is overcome by the installation of probes in the vicinity of each launcher. In ASDEX Upgrade a sniffer probe is either installed in the same sector or in a neighbouring sector, so far based on empirical analysis.

The assumption that the plasma acts as mode mixer is also supported by the diode protecting the electron cyclotron emission (ECE) system. This diode views the plasma along the ECE optics and does switch-off the ECRH in case a threshold is exceeded. 
Although the observation angle is very narrow (to allow high spatial resolution of the ECE diagnostic) the diode signal (also shown in Fig. 5) follows closely the signal of the sniffer probes.

As can be seen in the following section some arbitrariness in the fine tuning of the threshold level remains and may be due to a scenario dependent learning process. In any case the actual settings allow a safe immediate switch-off of the ECRH in case of cutoff or severely reduced single-pass absorption.

\section{Experimental results}

\subsection{Experiments with X3-mode heating}

Figure 6 shows the successful suppression of tungsten accumulation using X3 heating with an X2-beam dump at the plasma edge. Control parameters in both discharges
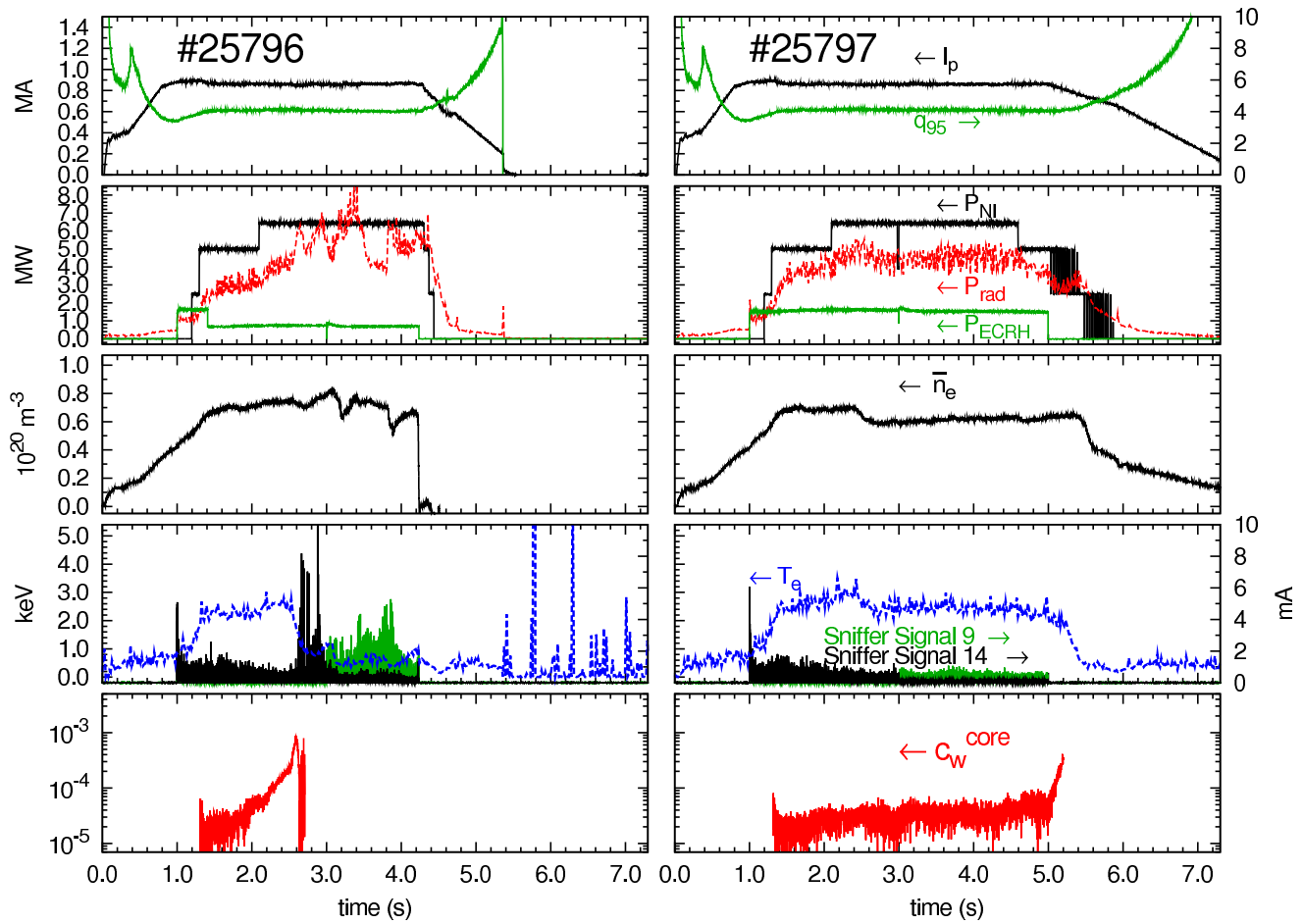

Figure 6. Identical X3 mode heated discharges differing only in the injected ECRH power. In the top plot the plasma current $I_{P}$ and the associated safety factor $q_{95}$ is plotted. In the second figure the heating power and the plasma radiation $P_{\text {rad }}$ (red curve) is shown. Here it can be seen that the ECRH power $P_{E C R H}=1 \mathrm{MW}$ (green line) in the discharge \#25796 was switch off in the beginning of the discharge due to arcing in the transmission line. A continuous rise in the tungsten concentration $c_{W}$ (bottom plot) is the consequence which leads to a high $P_{r a d}$ and to a decreasing $T_{e}$ (blue curve in the forth plot) in this discharge. Further parameters of the discharges were and are not plotted here: Greenwald density ratio $n / n_{G W}=0.7 ; \mathrm{H}$ factor $H_{98}=1.5$; triangularity $\delta=0.35$; maximal normalized beta $\beta_{N, \max }=3.5$; deuterium gas puff $=0.4 \cdot 10^{22} \mathrm{~s}^{-1}$. 


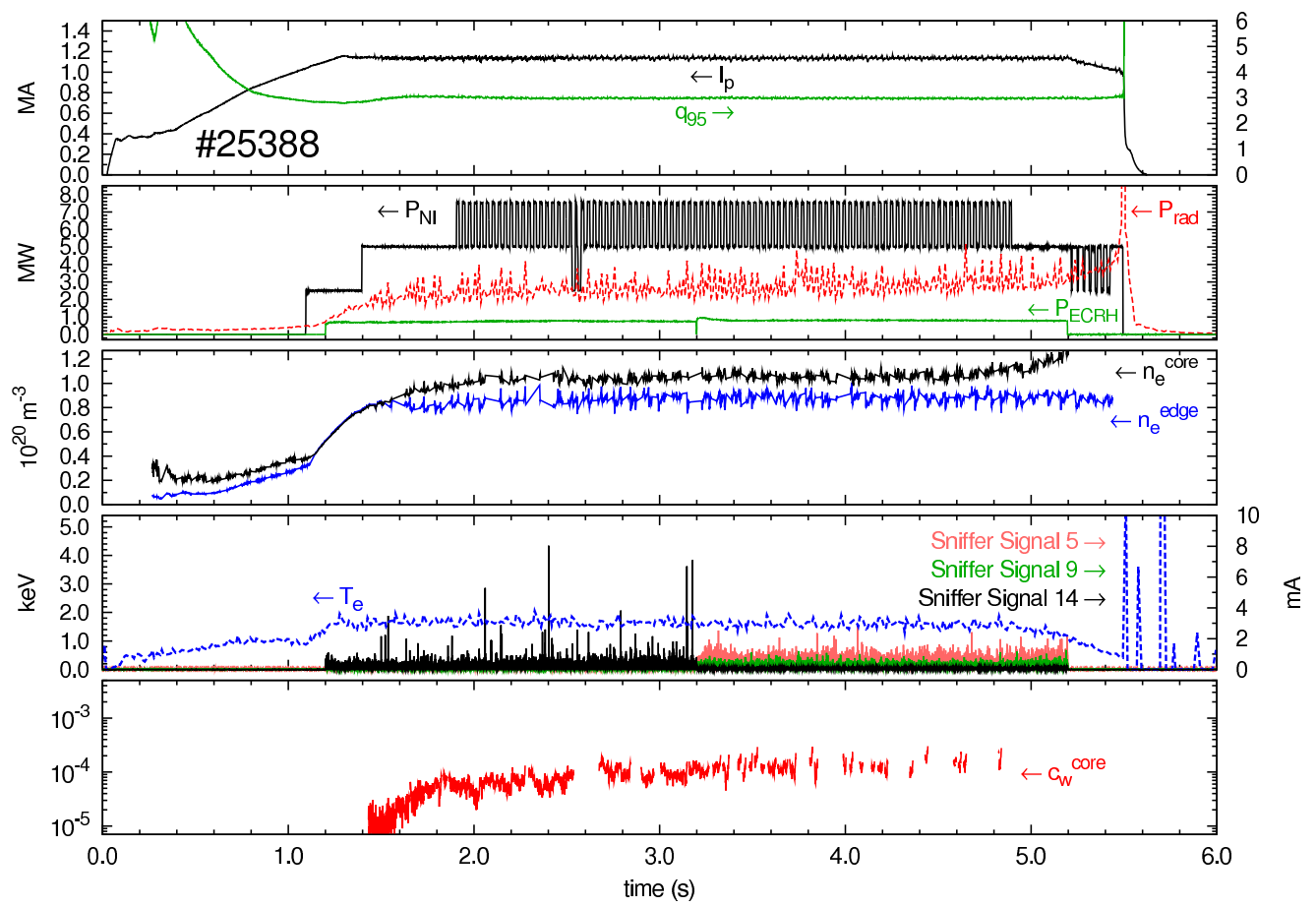

Figure 7. First $q_{95}=3$ discharge at tungsten coated ASDEX Upgrade with X3-mode heating with a higher $D$ gas puff of $0.9 \cdot 10^{22} \mathrm{~s}^{-1}$ (not shown here) than in the discharges in Fig. 6. The temperatures are in the range of $2-3 \mathrm{keV}$ and therefore the analysis of the tungsten concentration, needing temperatures of $\approx 3 \mathrm{keV}$ is not possible on the whole time trace. Nevertheless the tungsten did not accumulate in the core, but stays on a high level.

differ only by the amount of ECRH power. In the discharge on the left the ECRH power of $1 \mathrm{MW}$ was not sufficient to induce the needed electron temperature gradient to suppress the tungsten accumulation. The tungsten concentration $c_{W}$ rises and the electron temperature decreases due to higher radiation losses. At $2.7 \mathrm{~s}$ the temperature is reduced dramatically, such that the X2 resonance in the pedestal top cannot act as beam dump anymore, which induces a rise of the sniffer signal (forth plot in Fig 6). In contrast to this behaviour, the doubled ECRH power in \#25797 prevents the increase of the tungsten concentration in the core and stabilizes the plasma.

Due to the high heating power of NBI heating $P_{N I}=6.5 \mathrm{MW}$ and ECR heating $P_{E C R H}=1 \mathrm{MW}$ respectively $2 \mathrm{MW}$ in both discharges and the low magnetic field of $1.8 \mathrm{~T}$ the discharges are driven near the beta limit. Therefore a beta control has to be implemented in the future to prevent triggering of neoclassical tearing modes, which can be seen here at the density drop in discharge \#25797 at $2.5 \mathrm{~s}$.

However with higher gas puff it was also possible to suppress the tungsten accumulation with $1 \mathrm{MW}$. The gas puff influences the dominant tungsten source in the plasma edge, the ELMs, in such a way, that the ELM energy decreases causing a reduction of the tungsten influx [7]. As shown in Fig. 7 a discharge at $q_{95}=3$ and high confinement $\left(H_{98, y 2}=1.1\right.$ and $\left.n / n_{G W}=0.7\right)$, as expected for the ITER $Q=10$ scenario, 
was also successfully driven with a $D$ gas puff of $0.9 \cdot 10^{22}$ and 1 MW ECRH. Here the tungsten concentration stays on a high level $\left(c_{W}=1 \cdot 10^{-4}\right)$, but did not accumulate in the plasma core. However, the concentration is too high in view of a possible tungsten coated ITER, a reduction of this concentration to $c_{W}<5 \cdot 10^{-5}$ can be realized with an increased electron heating. Further experiments are foreseen in the next campaign.

\subsection{Test of the O2 reflector}

The new reflector tiles have to be tested with respect to the suitability of the embedded thermocouples and with respect to its capabilities in reflecting the non-absorbed power towards the plasma centre. The thermocouples were tested with high power microwaves of $0.4 \mathrm{MW}$ for $100 \mathrm{~ms}$ injected into the empty vacuum vessel of ASDEX Upgrade. In a scan over the whole surface of one mirror by changing the angles of the microwave launcher the behaviour of the thermocouples could be demonstrated. It was found that the thermocouples show a clear rise of their temperature $\left(\Delta T \approx 30{ }^{\circ} \mathrm{C}\right)$ only if the beam hits their direct neighbourhood (a few $\mathrm{cm}$ ) within a few $10 \mathrm{~ms}$ as shown in Fig. 8. Thus it is possible to use their signals for the localization of the microwave beam on the holographic mirrors.

The thermocouple signals of a discharge with plasma are shown in Fig. 9. The O2-mode beam (switched on in the grey region, the beam size can be compared to this in Fig. 8) was moved from above the mirror to the centre and back (launcher mirror movement is marked as violet line). The red (a) and green (b) curves belong to the thermocouples marked with crosses in the same colour. The monotone rise of the temperature of the thermocouples is due to the heating from the plasma so a comparison
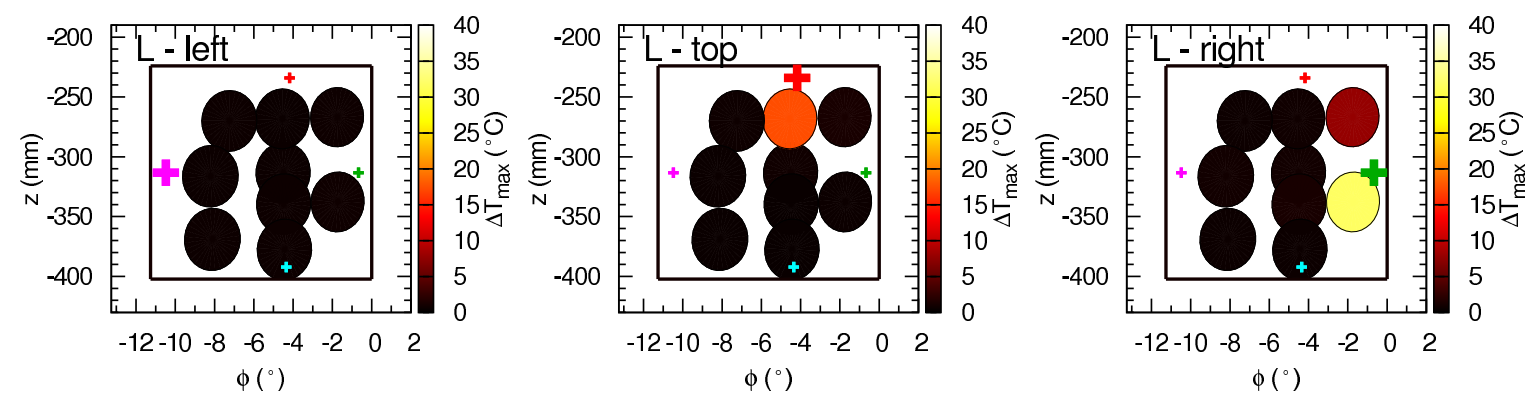

Figure 8. Test of the thermocouples on ECRH radiation. A scan of the beam over the whole surface of the mirror shows the dependence on the location of the beam. The spots show the locations of the beam on the mirror and the size of the circles represents the size of the beam. The colours of the spots indicate the temperature of the associated thermocouple (big cross in each plot). The bottom thermocouple was broken and is not shown here. Also the left one does not show a temperature rise on ECRH radiation. The different temperature response of the thermocouples on the ECRH radiation can be due to small inaccuracy during the installation process of the thermocouples in the mirrors. But these problems should be fixed with new and more thermocouples in the next campaign. 

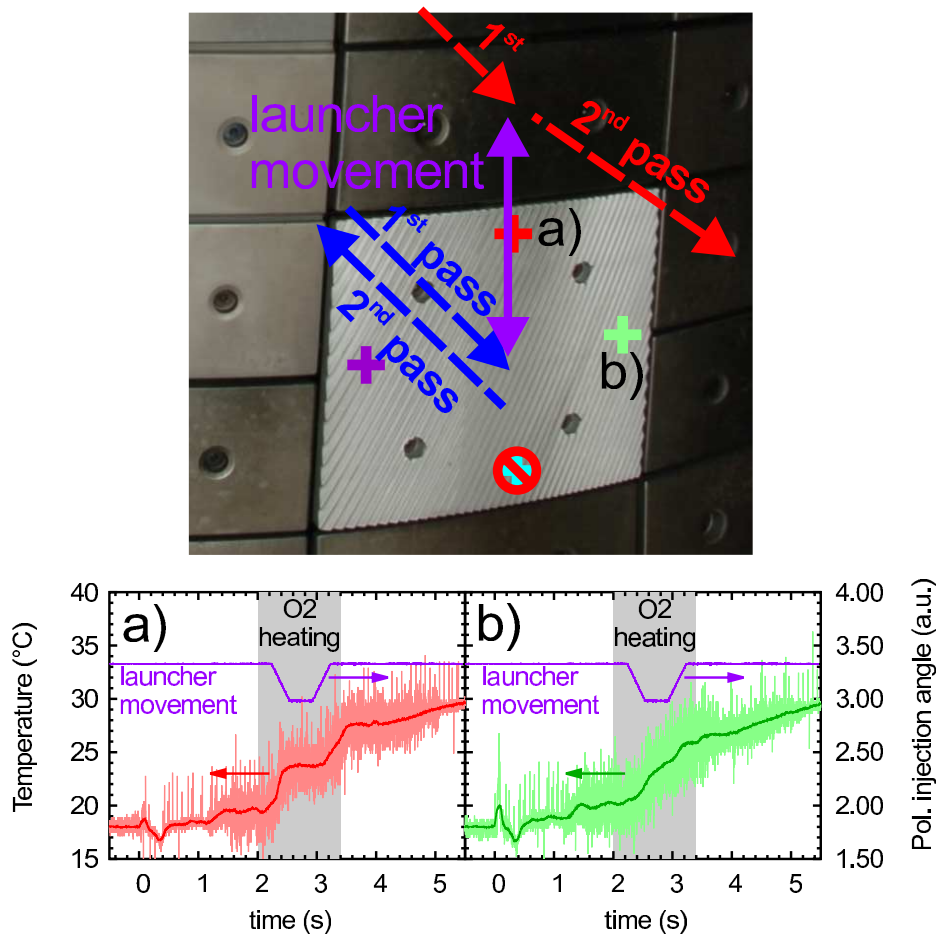

Figure 9. In the top picture the thermocouple test scenario with plasma is shown. The O2-mode beam was moved from above the holographic mirror to the centre of the mirror and back (violet solid line), the dashed arrows show the beam trajectories before and after the reflection on a standard tile (red) and on the holographic mirror (blue) (see also Fig. 10). The time traces of temperatures (raw data and $100 \mathrm{~ms}$ smoothed) of the thermocouples a) and b) are shown in the bottom picture, the red curve and the green curve belongs to the thermocouples in the same colour, the violet line shows the mirror movement and the grey region the O2-mode injection.

of two thermocouples is necessary to remove background heating of the plasma. The higher gradient in the temperature of the upper thermocouple in contrast to the left one during the movement of the beam seems suitable for a feedback control of the launcher angle in the future.

In the same discharge it was possible to show the effect of the reflector on the second pass of the O2-mode beam. During the O2-mode heating phase the power of the gyrotron was modulated with $30 \mathrm{~Hz}$. With a movement of the beam from a standard tile above the mirror to the centre of the mirror it was possible to compare directly the response of the electron temperature to a second pass.

A Fourier analysis of the electron temperature modulation is depicted in Fig. 10; the blue curve shows radial profiles of amplitude and phase of the temperature modulation with two passes (beam reflected on mirror) and the red curve with only one pass though the plasma core (beam reflected on a standard tile), see also Fig. 9. The plot can be interpreted in such a way: A higher absorption induces a higher modulation amplitude and a smaller cross phase between the power modulation signal and the electron temperature modulation. The heat waves travel out of the deposition region 


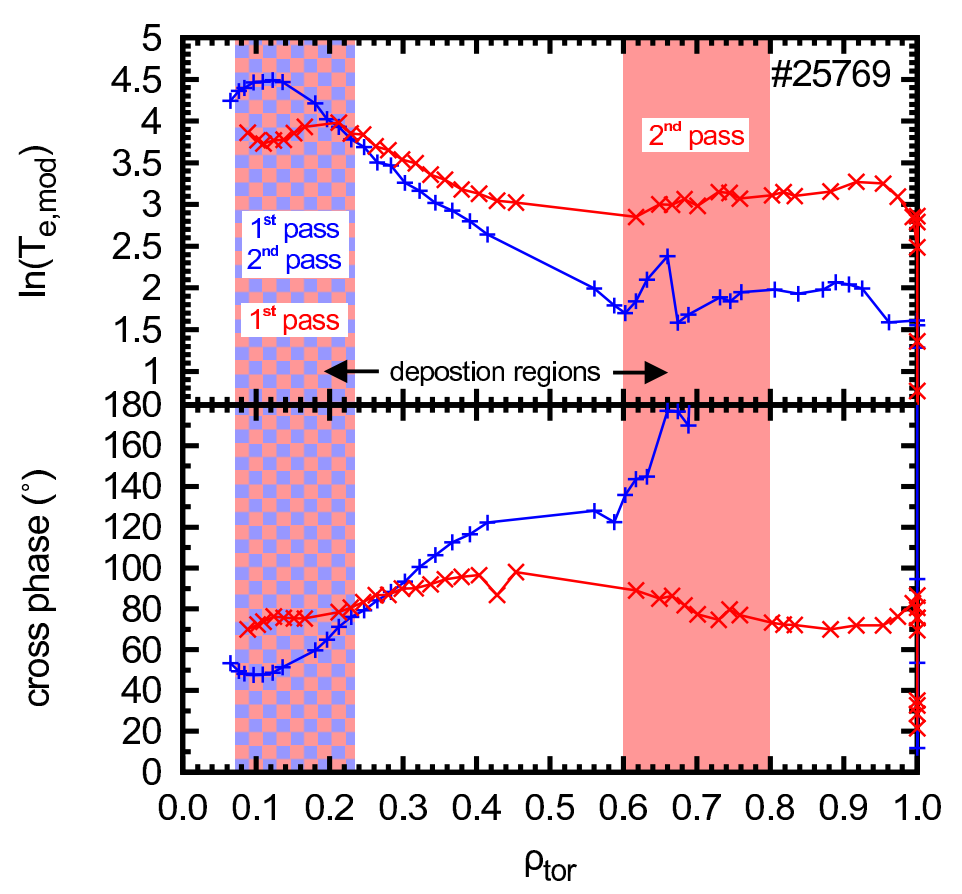

Figure 10. Amplitude and phase of the electron temperature modulation from a Fourier analysis as a function of the normalized toroidal flux radius $\rho_{t o r}$. The O2mode ECRH was on/off modulated with $30 \mathrm{~Hz}$, the blue curve shows the benefit of the second pass through the plasma centre in contrast to the reflection on a standard tile above the holographic mirror (for the heating geometry see also Fig. 9).

resulting in a smaller modulation amplitude and a higher cross phase [24]. Therefore for the dual pass through the plasma centre (deposition region is marked as blue/red crosshatched area) a higher modulation amplitude and a deeper cross phase than in single pass is expected and found in the modulation measurements. At the edge, the amplitude of the single-pass heated plasma is higher and the phase is lower than during the dualpass heating. This is a confirmation of the off-axis power deposition (red shaded area), which results from the reflection from a standard tile above the holographic mirror.

\subsection{Suppression of tungsten accumulation with O2 heating}

The tests in the previous section used plasmas with densities below the X2 cutoff and at least partially X2 heating, such that the tungsten accumulation was no issue. The new ECRH system was not available for most of the 2009 campaign, therefore, only preliminary experiments at the end of the campaign were possible. Figure 11 shows the first application of $\mathrm{O} 2$ heating in a high-triangularity, high-density discharge. Such discharges have to be run with nitrogen seeding to avoid overheating of the divertor [25]. In the first phase, the discharge is heated in X2 mode, which is switched off at $2.1 \mathrm{~s}$. O2 heating is switched on at $2.0 \mathrm{~s}$. About $0.2-0.3 \mathrm{~s}$ later the central tungsten concentration starts to rise, indicating that central heating is insufficient, similar to the discharge in Fig. 6 (left). Still we note that when changing from X2 to O2 heating 


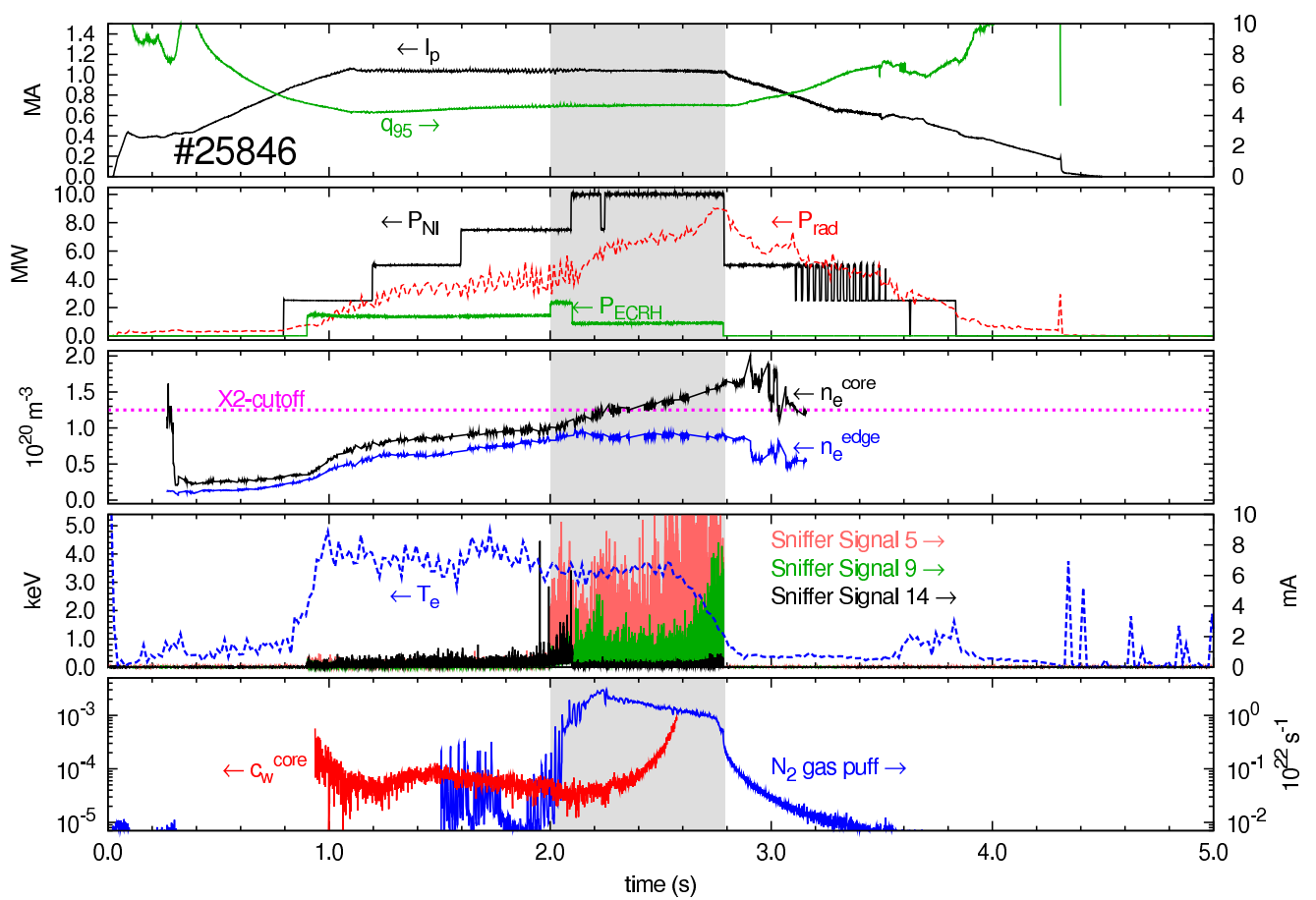

Figure 11. O2-mode heating of an X2-over dense plasma. The pattern region shows the time range where the $\mathrm{O} 2$ mode instead of $\mathrm{X} 2$ mode is injected. It can be seen, that $1 \mathrm{MW}$ are not sufficient to suppress the tungsten concentration. (Further plasma parameters: $n / n_{G W}>1 ; H_{98}=1.2 ; \delta=0.4 ; \beta_{N, \max }=2.5 ; D$ gas puff $=1.5 \cdot 10^{22} \mathrm{~s}^{-1}$ )

the central temperature does not drop. Also the central density exceeds the X2-cutoff density without a significant rise of the sniffer-probe signal. The late increase of the sniffer signal corresponds to a late decrease of $T_{e}$. Indeed this proves the suitability of the sniffer probe to detect the reduction of the optical thickness due to the drop of temperature.

We note here that also the nitrogen feedback was not optimal in this discharge. A strong $N_{2}$ puff was applied just between 2.1 and $2.3 \mathrm{~s}$ and this might have triggered the accumulation process.

Also the rising density changes the refraction index of the plasma resulting in a movement of the beam on the holographic mirror. TORBEAM simulations show a motion of the beam to the bottom of the reflector and perhaps out of it. Thus a loss of the second central absorption (20\% of the injected power) cannot be excluded. Unfortunately the bottom thermocouple was broken in this campaign so it was not possible to "see" the movement of the beam to the bottom. With more ECRH power and the real-time control of the fast steerable launcher, as foreseen for the next campaign, it should be possible to control the tungsten accumulation successfully also with $\mathrm{O} 2$ heating at a density above the X2 cutoff. 


\section{Conclusion and Outlook}

In this paper new ECR heating scenarios at ASDEX Upgrade where presented. With the O2- and the X3-mode heating, plasma parameters closer to the ITER target values can be achieved in ASDEX Upgrade. However, both modes suffer from incomplete absorption of the microwave.

Solutions to this problem have been presented for both cases: In the X3-mode heating scenario central heating with low tungsten concentration was obtained for $B_{t}(0)=1.75 \mathrm{~T}$, the shine-through can be absorbed at the $\mathrm{X} 2$ resonance on the high field side. First X3-mode heating experiments with the X2-beam dump at the plasma edge show a strong dependence of the tungsten concentration on the injected microwave power and on the gas puff level. In similar discharges only differing in the ECRH power the dependence of the tungsten accumulation on the injected microwave power was demonstrated. In the discharge with $1 \mathrm{MW}$ ECRH the tungsten concentration increased, leading to a radiation collapse. On the other hand with a higher gas puff it was shown that $1 \mathrm{MW}$ in X3 mode can be sufficient to control the impurities. In this discharge the ITER-like $q_{95}=3$ was obtained in the tungsten coated ASDEX Upgrade with high confinement and ECRH in X3 mode.

In the O2-mode heating scenario holographic mirrors provide a well defined second pass of the beam through the plasma centre and thus ensure a high absorption (up to $94 \%$ ). The benefit of the second pass through the plasma was demonstrated in modulation measurements. A first high-performance discharge with high triangularities and densities above the X2-mode cutoff was heated with the O2 mode and shows promising results. However, $2 \mathrm{MW}$ of ECRH power seem to be necessary for a robust suppression of tungsten accumulation in the regimes studied so far. This power is expected to be routinely available in the next experimental campaign.

The real-time beam-location system on the holographic mirror based on thermocouples will be ready in the next campaign, in order to react on refraction changes due to density variations in high-density plasma discharges.

\section{References}

[1] LeUterer, F., GRÜNWAld, G., MONACO, F., et al., Fusion Engineering and Design $\mathbf{7 4}$ (2005) 199 , Proceedings of the 23rd Symposium of Fusion Technology - SOFT 23.

[2] WAGneR, D., STOBER, J., LEUTERER, F., et al., Plasma Science, IEEE Transactions on 37 (2009) 395 .

[3] KALlENBACH, A., DUX, R., MAYER, M., et al., Nuclear Fusion 49 (2009) 045007.

[4] DUX, R., BOBKOV, V., HERRMANN, A., et al., Journal of Nuclear Materials 390-391 (2009) 858, Proceedings of the 18th International Conference on Plasma-Surface Interactions in Controlled Fusion Device, Proceedings of the 18th International Conference on Plasma-Surface Interactions in Controlled Fusion Device.

[5] Angioni, C., DUX, R., FABle, E., PeEters, A. G., and THE ASDEX UPGrade TEAm, Plasma Physics and Controlled Fusion 49 (2007) 2027.

[6] NEU, R., BOBKOV, V., DUX, R., et al., Journal of Nuclear Materials 363-365 (2007) 52 , Plasma-Surface Interactions-17. 
[7] DUX, R., NEU, R., PEETERS, A. G., et al., Plasma Physics and Controlled Fusion 45 (2003) 1815.

[8] GANDINI, F., CIRANT, S., HIRSCH, M., et al., Fusion Engineering and Design 56-57 (2001) 975 .

[9] ERCKMAnN, V. and GASPARINO, U., Plasma Physics and Controlled Fusion 36 (1994) 1869.

[10] BORNATICI, M., Plasma Physics 24 (1982) 629.

[11] POLI, E., PeEters, A. G., and PereverzeV, G. V., Computer Physics Communications 136 (2001) 90 .

[12] HÖHNLE, H., KASPAREK, W., STROTH, U., et al., O2- and X3-heating experiments on ASDEX Upgrade, in PRATER, R., editor, Proceedings of the 16th Joint Workshop on Electron Cyclotron Emission and Electron Cyclotron Resonance Heating, pp. 124-130, World Scientific, Singapore, 2010.

[13] HÖHNLE, H., KASPAREK, W., STOBER, J., et al., Investigation of the O2- and X3-mode heating in ASDEX Upgrade, in 36th EPS Conference on Plasma Phys. Sofia, June 29 - July 3, 2009 ECA Vol.33E, P-1.145, Sofia, 2009.

[14] PANkin, A., MCCUne, D., ANDRE, R., BAteman, G., and KRITZ, A., Computer Physics Communications 159 (2004) 157184.

[15] KRAUS, M., Heiz- und Stromprofile bei Neutralteilcheninjektion in Tokamakplasmen, Master's thesis, Technische Universität München, 2010.

[16] MANGOLD, O., KASPAREK, W., and HOLZHAUER, E., Optimization of microwave reflection gratings for electron cyclotron resonance heating in O2 mode, in Conference Digest of the 2004 Joint 29th International Conference on Infrared and Millimeter Waves and 12th International Conference on Terahertz Electronics, pp. 717 - 718, 2004.

[17] STORN, R., On the usage of differential evolution for function optimization, in Biennial Conference of the North American Fuzzy Information Processing Society, pp. 519 - 523, IEEE, 1996.

[18] MANGOLD, O., Effiziente holographische Gitterspiegel für die Elektronzyklotronresonanzheizung von Fusionsplasmen, $\mathrm{PhD}$ thesis, Universität Stuttgart, 2009.

[19] WAGNER, D., STOBER, J., LEUTERER, F., et al., Journal of Infrared, Millimeter and Terahertz Waves 32 (2011) 274, 10.1007/s10762-010-9703-3.

[20] MLYNEK, A., REICH, M., GIANNONE, L., et al., Nuclear Fusion 51 (2011) 043002.

[21] HICKS, N. K., SUTTROP, W., BEHLER, K., et al., Fusion Science and Technology 57 (2010) 1.

[22] GANDINI, F., private communication, 2008, ITER Organization.

[23] WAGNER, D., SCHMID-LORCH, D., STOBER, J., et al., Fusion Science and Technology 58 (2010) 658 .

[24] LeUterer, F., DUX, R., GANTEnBein, G., et al., Nuclear Fusion 43 (2003) 1329.

[25] KAllenBACH, A., ADAMEK, J., AHO-MAnTILA, L., et al., Nuclear Fusion , accepted for publication. 